\title{
Percutaneous Nephrostomy
}

\author{
Rameysh D. Mahmood, Lee Yizhi and Mark Tan M.L. \\ Dept of Diagnostic Radiology, Changi General Hospital \\ Singapore
}

\section{Introduction}

Percutaneous nephrostomy (PCN) is a passageway that is introduced percutaneously into the renal pelvicalyces that can later be maintained by a tube, stent or catheter. Following its introduction by Wickbom in 1954 who described percutaneous puncture of the renal pelvis as a diagnostic procedure, Goodwin and Casey first described its therapeutic use for relief of urinary tract obstruction the following year in 1955 (Goodwin, Casey et al. 1955; Stables, Ginsberg et al. 1978). Since then, this now commonplace procedure has undergone significant progress in both its technical and imaging aspects, with improvisation of puncture devices and techniques, coupled with the advancing imaging modalities used to guide the procedure. Thanks to its good safety profile, percutaneous nephrostomy is the preferred technique for treatment of various urological conditions, and its pioneering role for relief of urinary tract obstruction remains in good use until today.

This chapter aims to review the clinical use of percutaneous nephrostomy as well as the background technical aspects involved in carrying out the procedure. Some emphasis will be placed in the anatomical considerations that are crucial in determining approach as well as risk profile for an individual case. The associated known complications of the procedure will also be discussed, along with the therapeutic options available for the relevant complications.

\section{Indications and contraindications of percutaneous nephrostomy}

In essence, percutaneous nephrostomy may be performed for diagnostic or therapeutic purposes. For example, an antegrade pyelography can be performed following percutaneous nephrostomy to diagnose urinary tract obstruction. Its therapeutic uses on the other hand, can be seen to fall under two broad groups. Typically, the procedure is carried out to provide urinary diversion, and for a large number of cases this is related to urinary tract obstruction due to various causes. Secondly, the nephrostomy can be used to provide access to the urinary tracts for further intervention such as endopyeloscopy and nephrolithotomy. This is usually performed in collaboration with a urologist.

\subsection{Indications}

The following is a list of indications recognised by the Society of Interventional Radiology (SIR) (Ramchandani, Cardella et al. 2003): 
1. Provision of urinary diversion in cases of urinary tract obstruction, which may be secondary to intrinsic or extrinsic ureteral obstruction. This may be related to urinary calculi, malignancy or iatrogenic causes. The obstruction may be diagnosed incidentally on imaging studies, or patients may present with features of obstructive uropathy.

2. In cases of pyonephrosis, where there is urgency in providing immediate drainage as these patients are at risk of developing fulminant sepsis and shock. This may be suspected in patients with clinical features of sepsis, accompanied by flank pain and evidence of urinary tract obstruction on imaging.

3. Urinary diversion in cases of urinary leakage or fistula, which may in turn be related to trauma for example.

4. Urinary diversion for hemorrhagic cystitis.

5. Providing access for urological interventions and endoscopy (nephrolithotomy and removal of urinary calculus, ureteral stent placement, delivery of chemotherapeutic agents e.g. for upper tract transitional cell carcinoma, foreign body retrieval e.g. migrated ureteral stents). Percutaneous nephrostomy has been shown to provide adequate treatment of various types of urinary calculi including staghorn calculi.

The above indications can be applied to both native as well as transplanted kidneys.

\subsection{Contraindications}

Percutaneous nephrostomy has a good safety profile, and there is no single recognizable absolute contraindication (Ramchandani, Cardella et al. 2003). Relative contraindications however do exist, for which the benefits and potential risk must be weighed for each individual case.

Patients with known renal vascular malformations or arterial aneurysm are at risk of severe hemorrhage should there be accidental injury to these affected vessels. Nevertheless these patients may still require emergent decompression particularly in cases of urinary tract obstruction complicated by pyonephrosis. Careful preprocedural planning is vital, taking into account the nature of vascular malformations or aneurysm in detail by using the appropriate imaging method such as CT when determining approach and puncture tract. The performing physician should be aware of the potential need for angiographic embolization in these cases, particularly if bleeding becomes difficult to control and there is risk of hemodynamic instability. Similarly, patients with severe coagulopathy or bleeding diathesis are exposed to risks of severe hemorrhage. For these patients, thorough assessment of their coagulation profile as well as appropriate correction of coagulopathy may be necessary prior to the procedure.

Electrolyte imbalances such as severe hyperkalaemia may frequently be encountered particularly in cases of background chronic renal disease, and in whom the concomitant urinary tract obstruction may need to be urgently treated. In these cases, appropriate medical therapy is required to correct the electrolyte imbalance in order to reduce the risk of developing complications such as cardiac arrhythmia or cardioplegia (Ramchandani, Cardella et al. 2003).

Special attention should also be made to those patients with significant underlying morbidity or terminal illness who are deemed unsuitable for conventional surgery but yet there may be a role for percutaneous nephrostomy to provide a temporary measure. Risks of 
complications are higher in these patients, and they are ideally treated as an inpatient to ensure adequate planning prior to the procedure as well as providing periprocedural monitoring.

Fluoroscopic or CT-guided percutaneous nephrostomy may be contraindicated in pregnant patients in the first trimester in order to minimize radiation exposure to the fetus. Percutaneous nephrostomy performed using only ultrasound guidance has been described with good success rates (Gupta, Gulati et al. 1997; Ozden, Yaman et al. 2002), with Gupta reporting an overall success rate of $98.5 \%$. However minimum radiation exposure should always be borne in mind even in non-pregnant patients in accordance with ALARA (As Low As Reasonably Achievable) principle.

\section{Anatomical considerations}

Following assessment of the primary diagnosis and indication for percutaneous nephrostomy for each particular case, the procedure should not be performed without adequate review of all relevant imaging performed prior to the procedure. Percutaneous nephrostomy is usually performed using ultrasound or fluoroscopic guidance, although in many cases, CT may have been performed to arrive at the diagnosis prior to the procedure and correlation with these images may prove to be beneficial.

The primary diagnosis should be reviewed thoroughly, and this should include the cause and level of obstruction, degree of pelvicalyceal dilatation, as well as the most accessible renal calyx for catheter placement. If urinary calculi are present within the renal pelvis, their exact nature and location must be elucidated. The success rate for percutaneous nephrostomy has been reported to be lower in patients with a non-dilated collecting system, complex calculus disease and staghorn calculus (Ramchandani, Cardella et al. 2003). The kidney itself must also be assessed for the presence of anatomical variants or congenital anomalies such as horseshoe kidney.

Equally important to note is the vascular anatomy of the target kidney. Its precise delineation, as well as the presence of abnormal vascular malformations or aneurysmal dilatation should be noted. Injury to the first order segmental renal arteries may occur in the region of the renal pelvis, particularly if the puncture is made too medially. To prevent vascular injury and bleeding complications, the safest approach has been described by approaching the cusp of the papilla as far peripherally as possible, and by entering the kidney via the Brodel's line (Dyer, Regan et al. 2002). Brodel's line is a zone of relative avascularity and watershed territory, which is located just posterior to the lateral convex margin of the kidney, between the major anterior and posterior divisions of the renal artery. Care should be taken to avoid a through-and-through two-wall puncture of the renal pelvis as this runs the risk of injury to the anterior segmental renal artery.

The position of the affected kidney relative to the surrounding abdominal viscera should be thoroughly assessed as this has a bearing in determining the safest and most effective approach for renal puncture. Under normal circumstances, the posterolateral margins of the kidneys are immediately adjacent to the posterolateral aspects of the abdominal wall with no organs to interpose in between. Hence, a posterior approach is advantageous in avoiding the surrounding organs (Hruby 1990). Although the spleen, liver, pancreas and the adrenal glands are in close proximity to the kidneys, they are usually not shown to interpose 
between the posterior aspect of the kidney and the adjacent abdominal wall. Hruby described no injury to these organs in their retrospective review of 3100 patients who underwent percutaneous nephrostomy. However trans-splenic puncture has been reported in a series of patients who underwent percutaneous nephrostomy for nephrolithotomy (Carey, Siddiq et al. 2006).

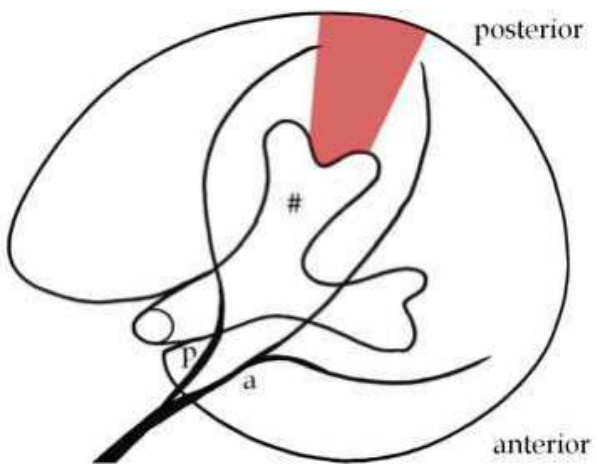

Fig. 1. Axial diagram of a kidney as seen in a prone patient illustrating the relations of the relatively avascular zone of Brodel (shaded) with the posterior $(\mathrm{p})$ and anterior (a) branches of the main renal artery as well as the posterior calyx (\#).

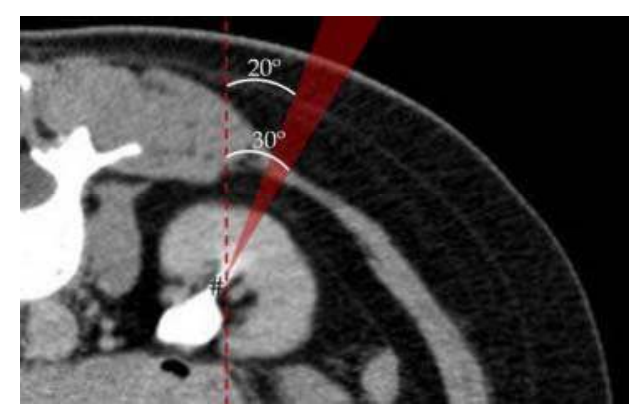

Fig. 2. The shaded wedge represents an ideal approach through Brodel's avascular zone. This approach of approximately $20^{\circ}-$ $30^{\circ}$ from the sagittal plane (dotted line) into the posterior calyx (\#) minimizes the risk of bleeding. A CT pyelogram is used to better illustrate the pelvicalyceal system.

There are exceptions to the above, as parts of the pleura lie in the posterior costodiaphragmatic recess that may overlap with the anterior pole of the kidney. Under normal circumstances, the lower line of the pleura usually crosses the $12^{\text {th }}$ rib at the lateral border of the erector spinae muscle, and part of the $12^{\text {th }}$ rib posterior to this point lies above the pleural line. This is important to note as a transpleural puncture may result in pneumothorax or hydrothorax, and for this reason, a subcostal approach should be used. Hruby described the best subcostal approach to be below the $12^{\text {th }}$ rib, approximately 2 fingerbreadth lateral to the lateral border of the paraspinal musclature, which is approximately along the posterior axillary line.

It is important to note however that the position of the kidneys in relation to the pleura varies according to respiration and individual anatomical variations, and this may be best assessed by using fluoroscopy just prior to puncture. The lower pole calyx is therefore the most likely to lie below the pleural line, and may in this way provide the safest approach. This is even more so in the right kidney, which is normally lower in position as compared to the left. However the upper pole calyx may have to be punctured in such cases where there is limited access to the lower pole calyx, for example due to presence of a large calculus. In such cases, a supracostal approach may have to be used with care.

In addition to the pleura, the colon is frequently found in close contact with the anteromedial aspect of the kidney, and too medial an approach may run the risk of colonic 
perforation. Occasionally, a retrorenal colon may also be encountered, and approach should therefore be negotiated accordingly. Although uncommon, cases of colonic perforation has been reported and this will be discussed further later in this chapter.

\section{Patient preparations, procedure and technique}

\subsection{Patient preparations}

As described, a patient who is about to undergo percutaneous nephrostomy should be thoroughly assessed for current physical status and presence of comorbidities that may affect the risk of developing complications following the procedure. Hyperkalaemia, should be corrected appropriately. Patients who are coagulopathic will have to be managed with plasma or platelet transfusion. Acceptable platelet and INR (International Normalised Ratio) levels vary between institutions, but INR values of less than 1.3 or platelet levels of more than 80,000/dL have been considered acceptable (Ramchandani, Cardella et al. 2003).

Prophylactic antibiotics have been widely used in preparing patients for percutaneous nephrostomy, although no clinical trial has published reports of its benefits to date. A prospective controlled study of patients undergoing percutaneous nephrolithotomy (Mariappan, Smith et al. 2006) reported significant reduction in the risk of upper tract infection and urosepsis following 1 week of prophylactic ciprofloxacin. However this may not be extrapolated in cases of percutaneous nephrostomy not related to underlying calculus or nephrolithotomy, as the presence of calculus is known to be associated with increased risk of infection. On a similar note, McDermott et al regarded the genitourinary tract as being contaminated in the presence of advanced age, diabetes, bladder dysfunction, indwelling urinary catheter, prior manipulation, ureterointestinal anastomosis, bacteriuria and calculi particularly of the struvite variety (McDermott, Schuster et al. 1997). This is particularly so in the presence of clinical signs of infection. It has been recommended that patients with low risk of infection receive a single dose of $1 \mathrm{~g}$ of intravenous cefazolin or ceftriaxone prior to the procedure (Ramchandani, Cardella et al. 2003). If these patients do not develop continuing signs of infection following the procedure, no further antibiotic treatment is necessary. Patients who are septic or with the above risk factors and at risk of developing infections, are recommended to prophylactically receive $1 \mathrm{~g}$ of intravenous ceftriaxone 8-hourly or 1g of IV sulbactame 6-hourly, along with 80mg of IV gentamycin 8hourly (Ramchandani, Cardella et al. 2003). Antibiotics are given for 5-7 days in the periprocedure period, and should be adjusted according to the results of urine culture obtained from the procedure.

Other aspects of patient preparation are common to most other interventional procedures performed in a hospital setting, and this entail obtaining informed consent regarding the procedure as well as adequate fasting if conscious sedation is considered. Certain groups of patients such as young children may have to undergo general anaesthesia, in which case collaboration with an anaesthetist may be necessary.

\subsection{Technique}

The patient is traditionally positioned in the prone or prone-oblique position, with the target puncture side elevated by approximately 20-30 degrees. The prone technique was originally adopted by Goodwin probably to avoid the colon and has since gained acceptance. The 
supine anterolateral position has also been recently suggested (Cormio, Annese et al. 2007) as being a safe and effective technique, with the benefits of greater patient comfort as well as causing less respiratory and circulatory difficulties in obese patients. Regardless, the target kidney should be reimaged and reassessed, and this is most commonly performed with ultrasonography. The target renal calyx should be identified and a planned approach should be clearly delineated. The puncture site should then be identified and marked at this stage of the procedure. As described above, the target renal calyx's position relative to the diaphragm during respiration should be observed, and ideally, a subcostal approach targeting Brodel's line should be utilized.

The site of renal puncture is determined by the indication for the procedure. A lower pole posterior calyx for instance, would be best used for simple urinary drainage (Dyer, Regan et al. 2002), while those of the upper and middle poles provide better access to the renal pelvis and ureter, especially if ureteral interventions are being considered. A puncture posterior to a calculus may assist in the treatment of calculus disease. These calyces are best identified by administration of intravenous iodinated contrast with visualization of contrast within the renal collecting system under fluoroscopic guidance. The anterior calyces are usually seen tangentially, while the posterior calyces are seen en face due to the orientation of the kidney about its horizontal axis. This would be contraindicated if the patient has prior history of contrast allergy or an underlying poor renal function, and it is probably not ideal in a severely obstructed system where poor contrast excretion can be expected.

After the patient has been adequately cleaned and draped using sterile methods, the puncture site should be infiltrated with an acceptable local anaesthesia such as $1 \%$ xylocaine. Instruction should be given to the patient to breathhold while a $21 \mathrm{G}$ diagnostic needle (e.g Accustick System - Boston Scientific, Neff Set - Cook Medical) is used to puncture the skin, which is then advanced posteroanterioly at an angle towards the intended calyx. Alternatively, a three-part co-axial needle may also be used, where there is an outer blunt cannula, an inner $22 \mathrm{G}$ needle as well as a stylet. As the renal fibrous capsule of the kidney is punctured, a finer needle may then be introduced via the coaxial needle to puncture the collecting system.

Movement of the needle that follows the kidney as the patient resumes respiration, as well as spontaneous drainage of urine from the needle as the needle stylet is removed, can be used to confirm successful renal entry. Spontaneous urine drainage is particularly seen in an obstructed system. If urine is not spontaneously draining, it may be aspirated from the needle instead. Sampled urine can be sent for culture and further analysis. Renal entry can be further confirmed by administration of contrast medium into the collecting system via the diagnostic needle.

A skin incision at the puncture site may now be performed, appropriately sized according to the catheter width that is to be used. A 0.018-inch guidewire is then passed through the needle to enter the renal pelvis (Figure 3). Over the guidewire, the tract is dilated to an appropriate size with a sheath/dilator assembly to later receive nephrostomy catheters, which can be up to $14 \mathrm{Fr}$ (French catheter scale) in size. The dilator and the 0.018 inch guidewire can now be removed, leaving the sheath in place (Figure 4). Subsequently, a 0.038inch guide-wire is advanced through the sheath and placed as distally into the ureter as possible to stabilize the tract (Figure 5). The nephrostomy catheter is then inserted over the guidewire (e.g. an 8Fr Navarre pig-tail catheter - Bard Nordic. Figures 6 and 7). The use of a 
metal cannula may be considered to stabilize the tube during its passage towards the kidney across the perirenal soft tissue.

Smaller-bore catheters $(7-8 \mathrm{Fr})$ are sufficient for drainage of non-infected and less viscid urine, while a larger-bore $14 \mathrm{Fr}$ catheter may be considered for drainage of infected urine or pus. Once the catheter is placed, its position can be further confirmed by administration of contrast to opacify the collecting system via the tube. The collecting system may be seen to decompress if the catheter is appropriately placed. Care should be taken to avoid over-distension of the collecting system so as to prevent bacteremia and risk of sepsis. To avoid catheter dislocation or dislodgement, self-retaining catheters should be used, and this should be placed as far into the collecting system as possible. Care must however be taken not to obstruct the ureter if a larger-bore catheter is used. Once firmly placed, the catheter is secured externally with retention sutures or other securing devices such as a skin disc.

Further urological intervention may follow the above puncture technique. The tract can be dilated further to allow passage of other instruments such as ureteroscope, balloondilatation system or nephrolithotomy instruments. A ureteral stent may also be placed through the percutaneous puncture. A larger-bore catheter may be considered by the urologist to allow for better drainage.

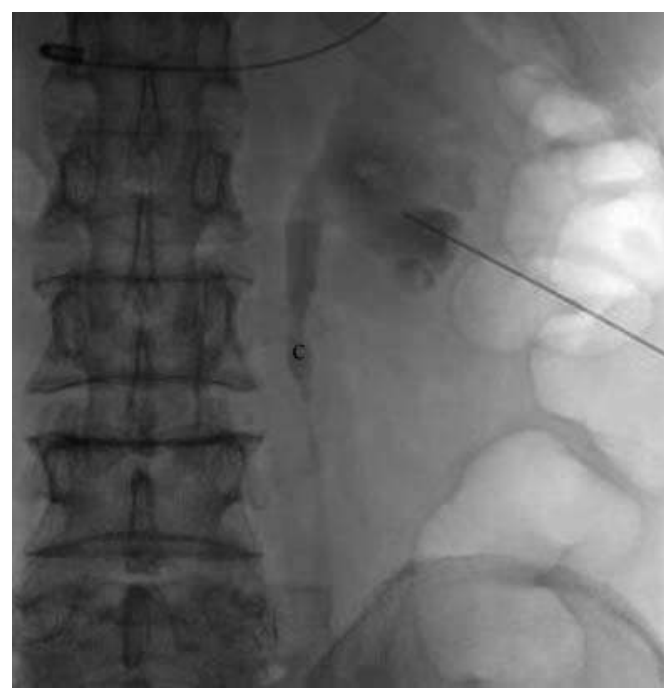

Fig. 3. A lower pole puncture was made with a 21 gauge Accustick needle into the lower pole calyx of the left kidney, with opacification of the collecting system by contrast. The obstructing calculus (c) can be seen along the proximal left ureter, causing upstream dilatation.

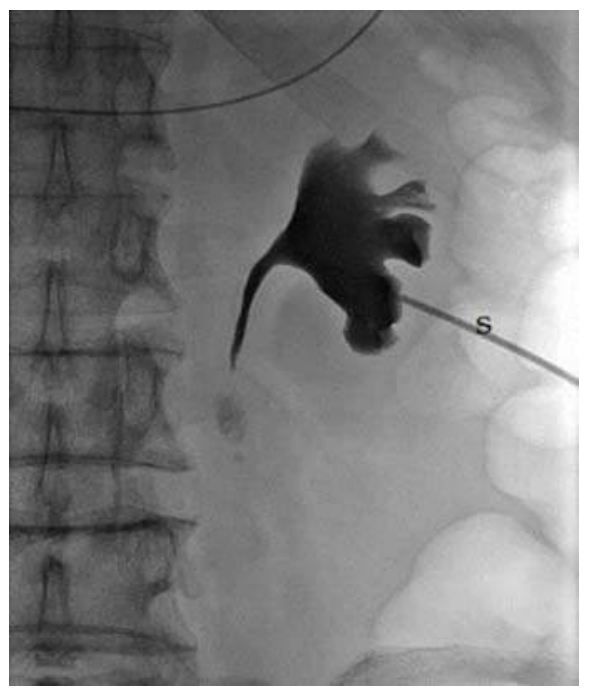

Fig. 4. A 0.018 inch guidewire was introduced through the needle into the renal pelvis as distally as possible. The sheath/dilator assembly was then advanced over the guidewire (not shown). The dilator and the 0.018-inch guidewire were then removed, leaving the sheath (s) in place. More contrast was instilled to delineate the collecting system. 


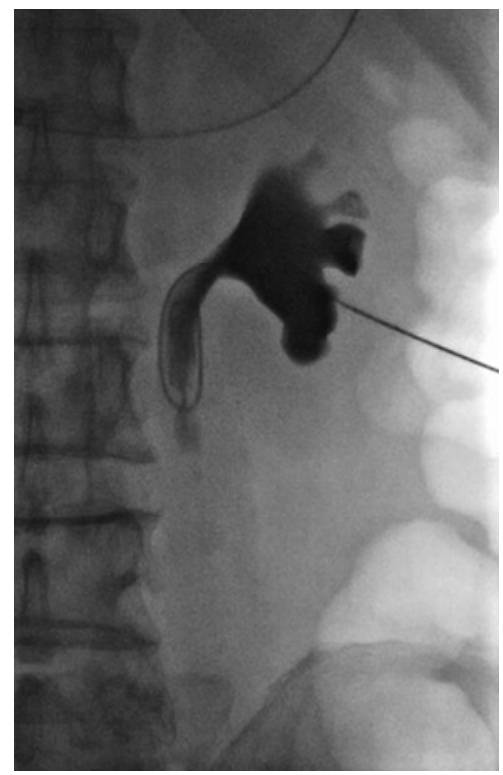

Fig. 5. Subsequently a 0.038 inch guidewire was advanced through the sheath and placed as distally into the ureter as possible for stability.

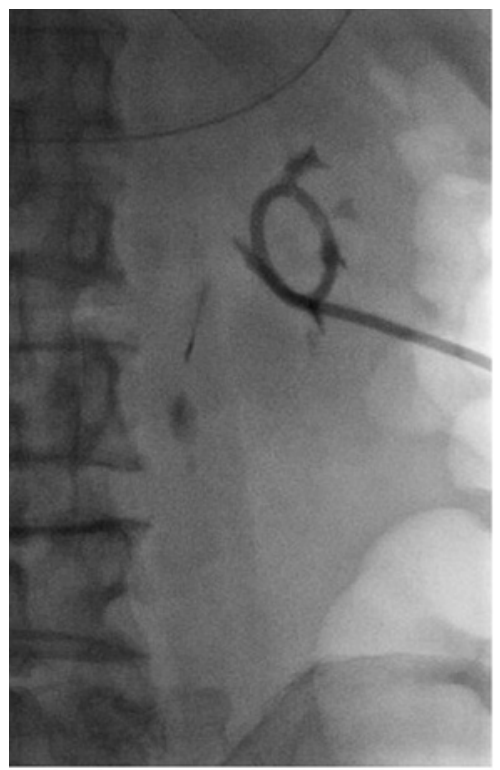

Fig. 6. An 8Fr Navarre catheter was then inserted over the 0.038 inch guidewire and secured in place.

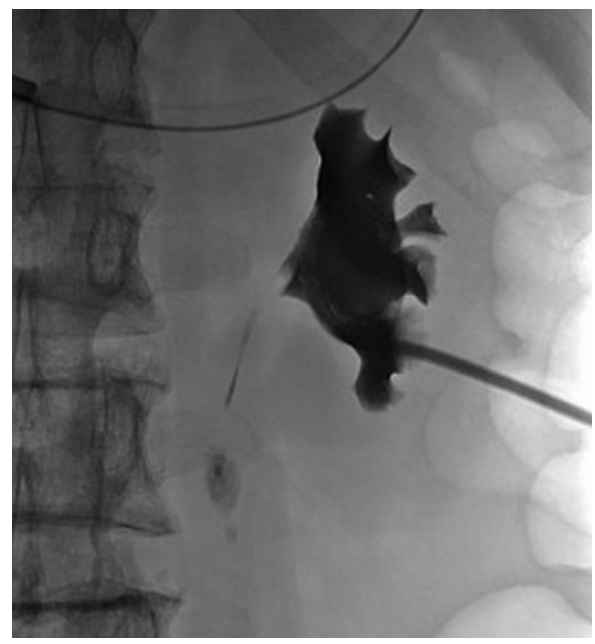

Fig. 7. The final image showing contrast opacification of the left renal collecting system. Note the reduced caliber of the upper ureter following successful drainage.

\subsection{Post-procedure care}

Post-procedure care is essential and may be crucial for early detection as well as reducing the risk of deterioration should complications occur during the procedure. High-risk 
patients may also require hospitalization for adequate monitoring. Frequent monitoring of vital signs should be routinely performed during initial recovery as signs of hemorrhage or sepsis may present suddenly and would require immediate attention. This should be accompanied by routine charting of the catheter output, noting the degree of hematuria as well as the output volume. Although commonly seen in virtually all patients, hematuria should resolve within 24-48 hours (Dyer, Regan et al. 2002). Prolonged hematuria should alert the physician to the possibility of persistent bleeding from vascular injury.

Catheter care is useful to reduce rate of catheter dislodgement and clogging, and it should be flushed with normal saline and aspirated routinely. Catheter clogging is a commonly occurring complication however, and this may even necessitate a change of catheter.

Antibiotics may be discontinued if there is low-risk of infection, although this should be continued in high-risk patients as described above. This should ideally be adjusted according to the urine culture results if available.

\section{Complications}

According to SIR, the reported success rate for percutaneous nephrostomy is $98-99 \%$, and this is defined as successful placement of catheter of sufficient size to allow for adequate drainage of the urinary tract or to allow successful tract dilatation for further interventional procedure. The success rates have been reported to be lower in cases of non-dilated collecting system or complex calculus disease (e.g. staghorn calculus) where a success rate of about $85 \%$ was reported (Ramchandani, Cardella et al. 2003). Despite the high success rates however, complications are frequently encountered, be it minor or major, with a reported incidence of approximately $10 \%$ of cases (Ramchandani, Cardella et al. 2003).

Several factors are associated with increased risk of complications. Patients at the extremes of age may develop complications from the procedure itself or even that related to the use of general anaesthesia, should this become necessary particularly in young children. Patient's coexisting comorbidities such as obesity, scoliosis, hepatomegaly and extremely mobile kidneys may necessitate greater manipulation, resulting in a technically challenging and thereby risky procedure. Further, in patients with chronic lung diseases and poor respiratory reserve such as emphysema, particular attention should be paid to the use of a subcostal approach to minimize risk of respiratory complications such as pneumothorax.

\subsection{Minor complications}

Minor complications are defined as complications occurring in relation to the procedure that are of no consequence and can be managed conservatively, or those requiring nominal therapy with no consequences (Ramchandani, Cardella et al. 2003). These patients may still require overnight hospitalization for observation. According to published reports, minor complications have been reported to occur in the range of 15-28\% of cases (Stables 1982; Lee, Smith et al. 1987; Dyer, Regan et al. 2002).

Post-procedure bleeding varies in severity, and may range from simple transient hematuria to severe hemorrhage requiring transfusion or intervention. Minor bleeding complications include transient hematuria, which occurs in virtually all patients, and small perirenal hematomas that can resolve on conservative management. Transient hematuria occurs very 
frequently that some authors do not regard it as a complication (Stables 1982). Clinically silent perinephric hematoma have been reported to occur fairly frequently, and is found in up to $13 \%$ of cases following percutaneous nephrostomy (Cowan 2008). These can resolve spontaneously without necessitating further interventions, leaving no serious consequences to the patient in the majority of cases. Stables also observed that in $79 \%$ of these cases, no significant renal alteration was seen. However, the presence of prolonged hematuria with or without hemodynamic instability should alert the physician for possible continuing bleeding as well as vascular injury.

Catheter-related complications such as kinking, obstruction or dislodgement may frequently be encountered and may require further intervention in $14 \%$ of cases (Cowan 2008). Published reports quoted varying rates of catheter dislodgement, from $4.8 \%-11.6 \%$. The use of larger bore catheters (for example 14Fr catheter) may reduce this rate to 1\% (Cowan 2008). Stables recommended advancement of the catheter well into the renal pelvis or calyces to minimize risk of dislodgement (Stables, Ginsberg et al. 1978). However care should be taken to avoid obstructing the ureter particularly if a large bore catheter is used. A dislodged tube may have to be replaced and the new catheter may have to be inserted by creating a new tract unless if the previous tract has been well established.

To reduce the rate of catheter obstruction, routine irrigation with normal saline solution should be performed after the procedure, although the use of larger-bore catheters may reduce the rate further while providing good drainage. Debris may also be removed by manipulation with a guide wire. Occasionally however, if catheter obstruction persists despite conventional measures, catheter replacement may be necessary.

Urine leak is known to occur following percutaneous nephrostomy, with a rate of approximately 7-7.2\% (Lee, Smith et al. 1987; Moskowitz, Lee et al. 1989). This is frequently minor, and contrast extravasation during or immediately after the procedure may or may not indicate ensuing complication. Also, most small leaks and tears resolve spontaneously with adequate urinary drainage or ureteral catheter insertion (Lee, Smith et al. 1987). Urine leak can also be controlled by using a larger bore catheter (Cowan 2008). However urine leak may occasionally be prolonged (lasting more than a week) and the ensuing urinoma may be large enough to require surgical intervention.

Other minor complications that may be seen following the procedure may include pain and fever. While fever can be worrisome for ensuing sepsis with potential of shock, febrile patients may require nothing more than conservative management with or without antipyretics. Lee reported $23 \%$ of raised temperature of more than 38.5 degrees Celsius in his published series of 582 patients who underwent percutaneous nephrolithotomy (Lee, Smith et al. 1987). These were attributed to retrograde urine flow as well as the use of irrigation fluid during the procedure. Minor wound infection has also been reported (von der Recke, Nielsen et al. 1994; Kaskarelis, Papadaki et al. 2001). These may be related to prolonged catheter use, and the use of sutures to secure the catheters to the skin (Kaskarelis, Papadaki et al. 2001). Pneumonia and atelectasis have been reported in a minority of cases, but is usually managed conservatively with antibiotics.

\subsection{Major complications}

Major complications are defined by SIR as complications that require therapy or minor hospitalization of up to 48 hours, as well as those that require major therapy, unplanned 
increase in level of care and prolonged hospitalization of more than 48 hours. Complications with permanent adverse sequelae or those that result in death are certainly considered to be major.

\subsubsection{Hemorrhage}

Hemorrhage requiring transfusion with or without radiological or surgical intervention is uncommon but is certainly a dreaded complication that carries a mortality risk. A number of published case series have reported major bleeding following percutaneous nephrostomy or percutaneous nephrolithotomy, and this occurs in the range of 1-4\% (Lee, Smith et al. 1987; von der Recke, Nielsen et al. 1994; Dyer, Regan et al. 2002; Ramchandani, Cardella et al. 2003). This may manifest in prolonged hematuria, hemodynamic instability and perirenal hematomas. Hemorrhage may be related to vascular injury during the procedure, whether a normal vessel that are inadvertently injured, or it may be related to underlying vascular malformations or aneurysm. Hemorrhage could also be attributable to an underlying coagulopathy or bleeding diathesis. The guideline for quality improvement by SIR recommended a threshold rate of hemorrhage requiring blood transfusion to be kept below $4 \%$.

Significant bleeding during the procedure may be controlled by a tamponade applied to the tract with a nephrostomy catheter or balloon dilatation catheter in larger tracts. If at any point that this fails, or if the patient develops subsequent significant blood loss after the procedure, angiographic evaluation would be indicated for identification of abnormal vascularity or major vascular injury with possible need for embolization. Surgical intervention may later become necessary if poor bleeding control is achieved. By this way, injured vessels may be ligated to arrest the bleeding, or as a last resort, partial or total nephrectomy may have to be performed. Lee reported 4 cases of persistent bleeding which were arrested by successful embolization, while a further 2 cases had to undergo nephrectomy or partial nephrectomy following failed embolization (Lee, Smith et al. 1987). Cowan reported 7 cases of persistent bleeding in a series of 3100 patients following percutaneous nephrostomy, and these were found to be secondary to underlying arteriovenous aneurysms that were treated successfully with embolization (Cowan 2008).

The performing physician should therefore be aware of the risk of severe blood loss, and the patient should be counseled appropriately regarding this risk during consent taking prior to the procedure. However, there are steps that can be taken during the procedure to minimise risk of hemorrhage. As described above, particular attention should be paid to the coagulation profile prior to the procedure, and any significant abnormality should be corrected accordingly. The renal vascular anatomy should be reviewed and taken into consideration when planning for puncture site and approach. The kidney should be punctured along the Brodel's avascular line as described above, and similarly, punctures too close to the inferior surface of a rib run the risk of injury to the intercostal vessels. The uses of fine needles and small-bore catheters have been associated with smaller risk of severe hemorrhage. A two-wall puncture of the renal pelvis should also be avoided to minimize risk of injury to the anterior segmental renal artery. As an additional support measure, highrisk patients should be prepared with support from the blood transfusion services should blood transfusion becomes necessary during or after the procedure. 


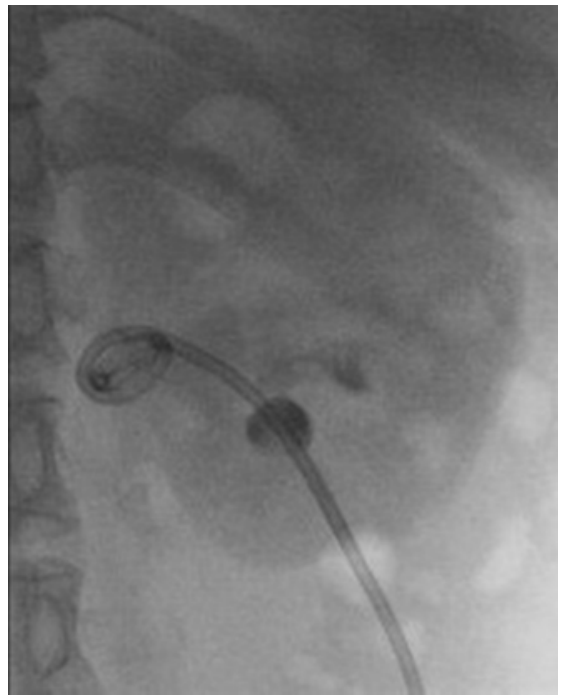

Fig. 8. An 8 Fr Navarre catheter in place with its loop apparently sited within the renal pelvis. Figures 8 to 11 are of the same patient.

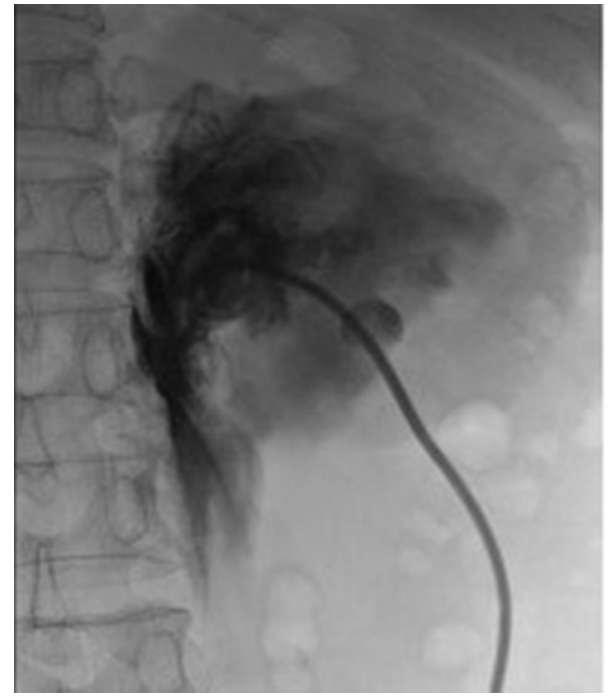

Fig. 9. Nephrogram showing contrast leakage into peripelvic fat due to transgression of the pelvicalyceal system. The patient subsequently developed flank pain and hypotension, indicating concomitant vascular injury. Sonography confirmed the presence of a perinephric hematoma.

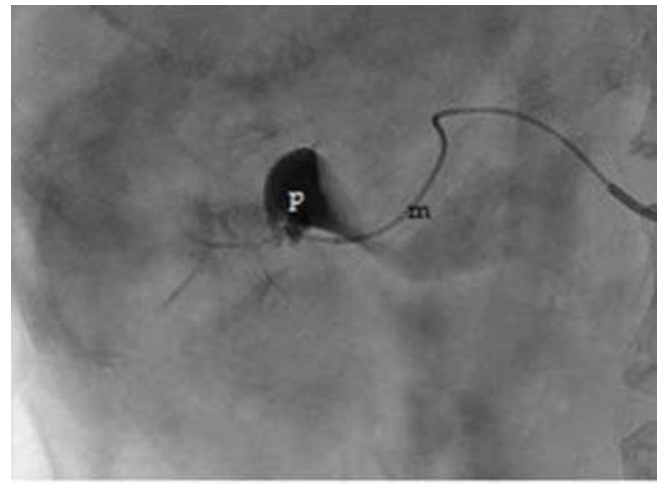

Fig. 10. Renal angiography demonstrated bleeding from a branch of the inferior segmental artery. Super-selective injection of contrast via a microcatheter $(\mathrm{m})$ into the bleeding artery showed extravasation into the renal pelvis $(\mathrm{p})$.

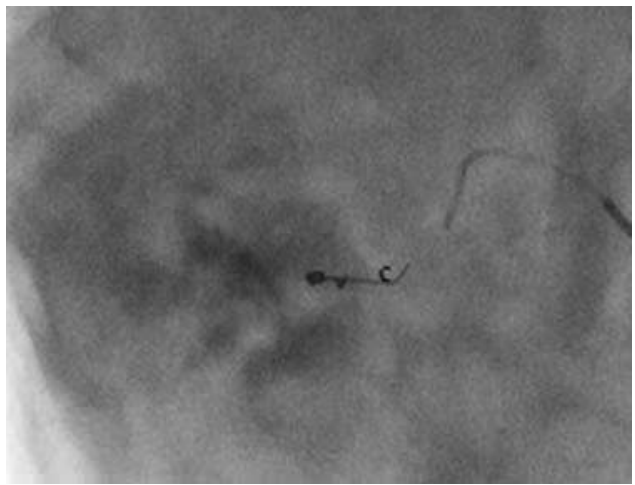

Fig. 11. Successful embolization with microcoils (c). 


\subsubsection{Sepsis}

Significant infection and sepsis following percutaneous nephrostomy is an important and well-recognised complication, with several published reports documenting its occurrence. According to SIR, sepsis related complications have been reported to occur in $1-9 \%$ of cases (Ramchandani, Cardella et al. 2003). There is a wide spectrum of severity of infection, but major sepsis may be defined as cases of septicemia requiring escalation in hospital care and longer use of antibiotics therapy, with or without shock. Transient fever is common following the procedure, occurring in almost all patients, the majority of which may settle in less than 6 hours (Lee, Patel et al. 1994). However, persistent fever with chills and signs of hemodynamic instability are worrisome signs and should be identified and treated accordingly. Septic shock is a serious complication, and has been reported to be a contributing factor towards patients mortality in some published case series. In 318 patients who underwent percutaneous nephrostomy, Lewis reported sepsis as the most common major complication, occurring in $2.2 \%$ of patients in his published case series, and it is the most severe complication necessitating intensive care (Lewis and Patel 2004). Sepsis was also considered to be a contributing factor in the death of two of these cases. Moskowitz further reported 2 cases of septicemia with shock in 11 cases of severe sepsis in his published case series of patients who underwent percutaneous nephrolithotomy (Moskowitz, Lee et al. 1989). SIR recommended a rate of septic shock of less than $4 \%$, and a rate of less than $10 \%$ for cases of septic shock in the setting of pyonephrosis.

Several factors have been found to contribute towards increased risk of sepsis, and this includes the duration of the procedure itself, urine bacterial load, severity of urinary tract obstruction as well as presence of bacteria within the calculus (Mariappan, Smith et al. 2006). Mariappan also reported higher risk of upper urinary tract infections in patients with calculi larger than $20 \mathrm{~mm}$ or a dilated pelvicalyceal system. Further, the puncture itself, and even the removal of calculus may reactivate underlying pre-existing infection within the urinary tract with release of bacteria into the system. Therefore, care should be taken to avoid over-distension of the renal collecting system during puncture, as this may result in bacterial reflux into the peripapillary plexus. Urine extravasation and absorption of irrigation fluid have also been found to be contributory (Lee, Patel et al. 1994).

The use of prophylactic antibiotics is therefore recommended in high-risk patients, and this has been shown to be of some benefit as reported in a prospective controlled study by Mariappan, who prescribed one week of ciprofloxacin to patients prior to percutaneous nephrolithotomy (Mariappan, Smith et al. 2006). Patients who received prophylactic ciprofloxacin were reported to have significantly reduced incidence of upper urinary tract infection as compared to the control group, with three times less risk of developing systemic inflammatory response syndrome. Antibiotics therapy may be further escalated in patients with evidence of urosepsis following the procedure, and this is best adjusted according to the results of urine culture and sensitivity.

\subsubsection{Pleural complications}

Pleural complications such as pneumo-, hydro-, or hemothorax and empyema are uncommon but have been known to occur from percutaneous nephrostomy, with a reported rate of $0.1-0.3 \%$ (Dyer, Regan et al. 2002; Ramchandani, Cardella et al. 2003). The risk of 
pneumo- and hydrothorax is reported to be in the range of $4-12 \%$ if a supracostal approach is used for puncture of the renal upper pole (Carey, Siddiq et al. 2006), although this may be difficult to avoid if it provides the best access to the collecting system. The use of a working sheath is an important consideration in these cases, as it may prevent leakage of fluid into the pleural cavity along the pleural tract during the procedure. Although pleural complications may be treated conservatively (Dyer, Regan et al. 2002), pleural drainage with chest tube insertion may be necessary.

\subsubsection{Bowel transgression and colonic perforation}

Bowel transgression is another uncommon but potentially serious complication of percutaneous nephrostomy, and is reported to occur in $0.2-0.3 \%$ of cases (Ramchandani, Cardella et al. 2003; M Tan 2010). Several risk factors have been recognized that may contribute to increased risks. Patients with a markedly dilated collecting system, colonic obstruction and patients with scarce perirenal fat are more likely to have a more posteriorly located colon. This increases the risk of colonic transgression when approaching the kidney. An anatomical variant to note is the retrorenal colon which is reported to occur in $1-1.9 \%$ of supine patients and in up to $10-16 \%$ of prone patients (Hopper, Sherman et al. 1987; Tuttle, Yeh et al. 2005). This retroperitoneal bowel loop is usually gas-distended and is found mostly around the lower renal poles. Care should thus be taken to visualize this with fluoroscopy or CT before any invasive percutaneous renal procedure. Colonic perforation has also been associated with right upper calyceal punctures in patients with horseshoe kidneys (El-Nahas, Shokeir et al. 2006). Any factor contributing to poor visualization of the kidneys during image-guidance such as gross obesity, abundance of gas-filled bowel loops and mobile kidneys may also result in inadvertent colonic injury. The risk is further increased when too lateral an approach is used to puncture the kidney (Wah, Weston et al. 2004).

Most cases of reported colonic perforation due to percutaneous nephrostomy are retroperitoneal and contained, and these have been managed well conservatively with good recovery (Wah, Weston et al. 2004). However, surgical repair may be required in cases of intraperitoneal colonic perforation, or where there is ensuing hemorrhage with or without shock. M Tan described a case of inadvertent colonic injury during percutaneous nephrostomy that occurred in a thin middle-aged woman with a dilated renal pelvicalyces (M Tan 2010). The patient was asymptomatic and the perforation was only discovered 3 weeks later during a double J stent insertion when contrast was noted in the colon. The patient was managed conservatively and the percutaneous nephrostomy was later withdrawn into the colon, functioning as a percutaneous colostomy. The use of antibiotic cover would be indicated in these cases to prevent infection.

The use of image guidance is important in reducing risk of colonic perforation. The exact location of the colon relative to the kidney should be identified prior to the procedure, and as described above, too lateral an approach should be avoided in high-risk patients. In patients with risk factors leading to poor visualization of the urinary system under ultrasound guidance, CT scan should be used to look for anatomical variants, such as a retrorenal colon or horseshoe kidney, to reduce the chance of inadvertent colonic puncture (M Tan 2010). 


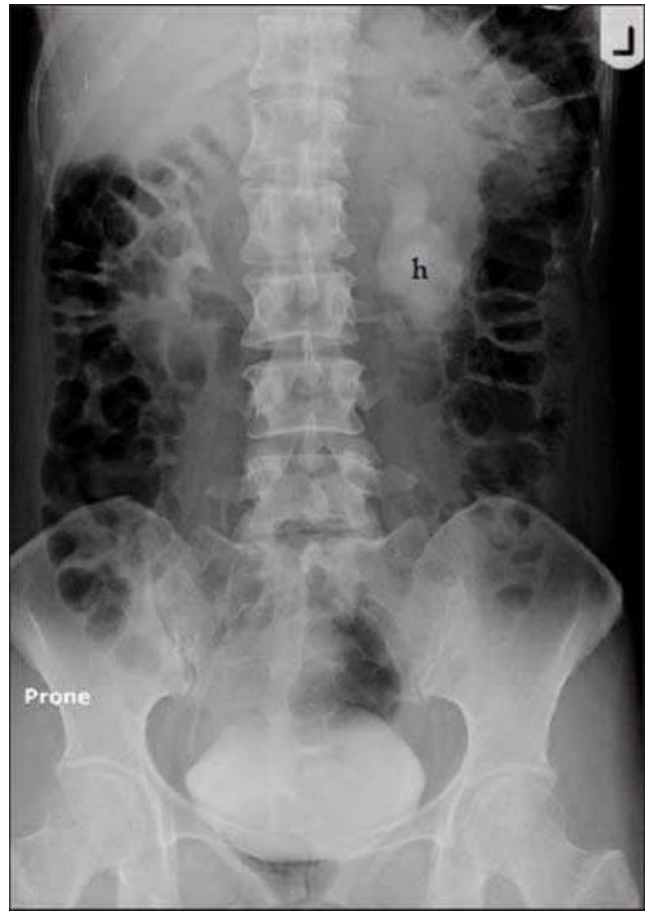

Fig. 12. Delayed phase of an intravenous urogram showing left hydronephrosis $(\mathrm{h})$ due to obstruction at the pelviureteric junction. The gas-distended descending colon is in close proximity to the lateral aspect of the left kidney.

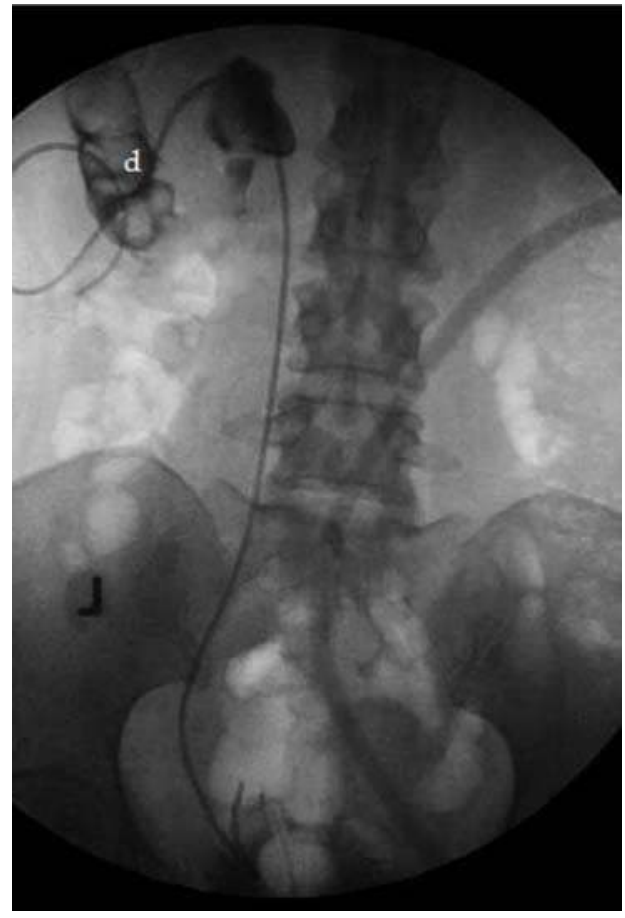

Fig. 13. Anterograde insertion of a double J stent 3 weeks later on the same patient showed extravasation of contrast through the percutaneous nephrostomy tract into the descending colon (d).

\subsubsection{Injury to intra-abdominal viscera}

Injuries to organs adjacent to the kidneys have been reported in less than $1 \%$ of cases (M Tan 2010), and of these, splenic injury is the most commonly reported. Liver laceration is less common, and seldom requires intervention (Lee, Smith et al. 1987).

The risk of splenic injury is increased if a higher supracostal approach $\left(10^{\text {th }}-11^{\text {th }}\right.$ ribs $)$ is used, or if the approach is made during inspiration. Should a trans-splenic tract is made, the primary concern is that of hemorrhage with risk of shock, and these may have to be managed surgically. However, conservative management may be considered in selected cases, particularly if the patient is asymptomatic and stable, and this was reported by Carey in a patient who sustained splenic injury that occurred during percutaneous nephrolithotomy (Carey, Siddiq et al. 2006). The patient was managed conservatively, with no serious consequences and the patient was discharged following removal of the nephrostomy catheter. 


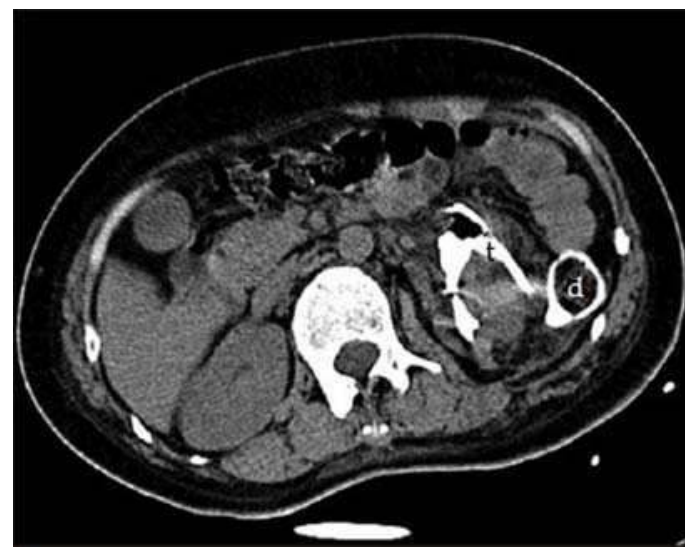

Fig. 14. Computed tomography scan showing extravasation of contrast from the dilated left collecting system through the left PCN tract ( $t)$ into the descending colon (d). In this case the tract had matured without any appreciable extravasation of contrast into the retroperitoneal space.

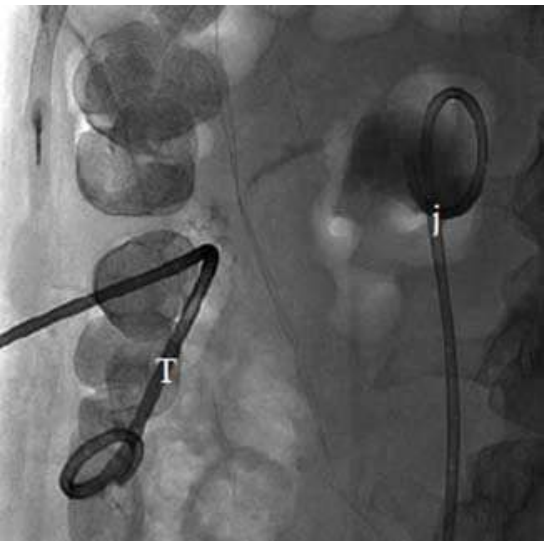

Fig. 15. Withdrawal of the PCN into the colon to be used as a percutaneous colostomy tube $(\mathrm{T})$ was performed after confirmation of good anterograde urinary drainage via the double-J stent (j).

Subsequent tube review confirmed closure of the colorenal fistula.

\subsubsection{Death}

Percutaneous nephrostomy has a low mortality rate, with published data reporting rates of $0.03 \%$ (Hruby 1990) and 0.3\% (Lee, Smith et al. 1987). Various major complications may contribute to death following the procedure, particularly in relation to severe hemorrhage and sepsis, but it may also be contributed by other complications provoked by the procedure itself. Myocardial infarction and cardiac arrest have been reported (von der Recke, Nielsen et al. 1994). Lee reported deaths in 2 patients, one of which was attributed to respiratory failure related to underlying severe interstitial pulmonary fibrosis, while the other was due to myocardial infarction in an obese diabetic patient with hypertension. The presence of comorbidities is therefore an important predisposing factor. Patients who require general anesthesia may also be at risk of developing associated complications. However the mortality rate for percutaneous nephrostomy remains lower than conventional surgery for patients who require urological intervention but are not good candidates for conventional surgery (Lee, Patel et al. 1994).

\section{Role of percutaneous nephrostomy in transplanted kidneys}

The indications for percutaneous nephrostomy described above can be similarly adopted for transplanted kidneys, and indeed percutaneous nephrostomy has been shown to have a good safety profile in these cases (Mostafa, Abbaszadeh et al. 2008). Mostafa further demonstrated that there was no statistical difference in the 10-year survival rates of renal transplant recipients who underwent percutaneous nephrostomy when compared to other renal transplant recipients without urological complications. It also serves as a useful alternative to conventional surgery, which may pose a higher risk in these patients. 
The most common urological complications in transplanted kidneys are ureteral obstruction and leakage (Mostafa, Abbaszadeh et al. 2008). These should be recognized and treated early to prevent graft failure. Ureteral obstruction is most commonly due to stricture at the ureterovesical junction anastomosis, brought about by fibrosis secondary to ischemia or rejection and therefore presents late. Mostafa reported good success rates in the treatment of these strictures, by using stents and balloon dilatations inserted via the percutaneous nephrostomy tracts. Early ureteral obstruction on the other hand may be related to other factors such as blood clots, calculus, edema or ischemic necrosis. Similarly, percutaneous interventions may be performed in the treatment of these cases.

\section{Conclusion}

Percutaneous nephrostomy is a widely used urological procedure, providing urinary diversion and access to the urinary tracts for other interventions. While demonstrating a good safety profile, many aspects of the procedure are associated with risks of complications, which may be contributed by various factors from the moment the patient is prepared until after the procedure. The performing physician must not only be well versed with the techniques involved, but he or she should also be well acquainted with the associated risks and complications so that these may be detected and treated early.

\section{References}

Carey, R. I., F. M. Siddiq, et al. (2006). Conservative management of a splenic injury related to percutaneous nephrostolithotomy. JSLS 10(4): 504-506.

Cormio, L., P. Annese, et al. (2007). Percutaneous nephrostomy in supine position. Urology 69(2): 377-380.

Cowan, N. (2008). The Genitourinary Tract; Technique and Anatomy. Grainger E Allison's Diagnostic Radiology, A Textbook of Medical Imaging. A. K. D. A. Adam, Churchill Livingstone. 1: 813-822.

Dyer, R. B., J. D. Regan, et al. (2002). Percutaneous nephrostomy with extensions of the technique: step by step. Radiographics 22(3): 503-525.

El-Nahas, A. R., A. A. Shokeir, et al. (2006). Colonic perforation during percutaneous nephrolithotomy: study of risk factors. Urology 67(5): 937-941.

Goodwin, W. E., W. C. Casey, et al. (1955). Percutaneous trocar (needle) nephrostomy in hydronephrosis. J Am Med Assoc 157(11): 891-894.

Gupta, S., M. Gulati, et al. (1997). Percutaneous nephrostomy with real-time sonographic guidance. Acta Radiol 38(3): 454-457.

Hopper, K. D., J. L. Sherman, et al. (1987). The variable anteroposterior position of the retroperitoneal colon to the kidneys. Invest Radiol 22(4): 298-302.

Hruby, W. (1990). Percutaneous Nephrostomy. Interventional Radiology. P. R. Robert F. Dondelinger, Jean Claude Kurdziel, Sydney Wallace, Thieme: 234 - 244.

Kaskarelis, I. S., M. G. Papadaki, et al. (2001). Complications of percutaneous nephrostomy, percutaneous insertion of ureteral endoprosthesis, and replacement procedures. Cardiovasc Intervent Radiol 24(4): 224-228.

Lee, W., A. Smith, et al. (1987). Complications of percutaneous nephrolithotomy. Am. J. Roentgenol. 148(1): 177-180. 
Lee, W. J., U. Patel, et al. (1994). Emergency percutaneous nephrostomy: results and complications. J Vasc Interv Radiol 5(1): 135-139.

Lewis, S. and U. Patel (2004). Major complications after percutaneous nephrostomy-lessons from a department audit. Clin Radiol 59(2): 171-179.

M Tan, P. u., PS Jaywantraj, D Wong (2010). Colonic Perforation during Percutaneous Nephrolithotomy Treated Conservatively. J HK Coll Radiol. 12(3): 117-121.

Mariappan, P., G. Smith, et al. (2006). One week of ciprofloxacin before percutaneous nephrolithotomy significantly reduces upper tract infection and urosepsis: a prospective controlled study. BJU Int 98(5): 1075-1079.

McDermott, V. G., M. G. Schuster, et al. (1997). Antibiotic prophylaxis in vascular and interventional radiology. AJR Am J Roentgenol 169(1): 31-38.

Moskowitz, G. W., W. J. Lee, et al. (1989). Diagnosis and management of complications of percutaneous nephrolithotomy. Crit Rev Diagn Imaging 29(1): 1-12.

Mostafa, S. A., S. Abbaszadeh, et al. (2008). Percutaneous nephrostomy for treatment of posttransplant ureteral obstructions. Urol J 5(2): 79-83.

Ozden, E., O. Yaman, et al. (2002). Sonography Guided Percutaneous Nephrostomy: Success Rates According to the Grade of the Hydronephrosis. Journal of Ankara Medical School 24(2): 69-72.

Ramchandani, P., J. F. Cardella, et al. (2003). Quality improvement guidelines for percutaneous nephrostomy. J Vasc Interv Radiol 14(9 Pt 2): S277-281.

Stables, D. P. (1982). Percutaneous nephrostomy: techniques, indications, and results. Urol Clin North Am 9(1): 15-29.

Stables, D. P., N. J. Ginsberg, et al. (1978). Percutaneous nephrostomy: a series and review of the literature. AJR Am J Roentgenol 130(1): 75-82.

Tuttle, D. N., B. M. Yeh, et al. (2005). Risk of injury to adjacent organs with lower-pole fluoroscopically guided percutaneous nephrostomy: evaluation with prone, supine, and multiplanar reformatted CT. J Vasc Interv Radiol 16(11): 1489-1492.

von der Recke, P., M. B. Nielsen, et al. (1994). Complications of ultrasound-guided nephrostomy. A 5-year experience. Acta Radiol 35(5): 452-454.

Wah, T. M., M. J. Weston, et al. (2004). Percutaneous nephrostomy insertion: outcome data from a prospective multi-operator study at a UK training centre. Clin Radiol 59(3): 255-261. 


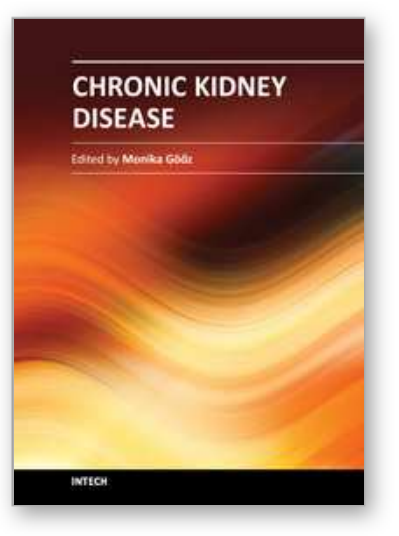

\author{
Chronic Kidney Disease \\ Edited by Prof. Monika Göőz
}

ISBN 978-953-51-0171-0

Hard cover, 444 pages

Publisher InTech

Published online 16, March, 2012

Published in print edition March, 2012

Chronic kidney disease is an increasing health and economical problem in our world. Obesity and diabetes mellitus, the two most common cause of CKD, are becoming epidemic in our societies. Education on healthy lifestyle and diet is becoming more and more important for reducing the number of type 2 diabetics and patients with hypertension. Education of our patients is also crucial for successful maintenance therapy. There are, however, certain other factors leading to CKD, for instance the genetic predisposition in the case of polycystic kidney disease or type 1 diabetes, where education alone is not enough.

\title{
How to reference
}

In order to correctly reference this scholarly work, feel free to copy and paste the following:

Rameysh D. Mahmood, Lee Yizhi and Mark Tan M.L. (2012). Percutaneous Nephrostomy, Chronic Kidney Disease, Prof. Monika Göőz (Ed.), ISBN: 978-953-51-0171-0, InTech, Available from: http://www.intechopen.com/books/chronic-kidney-disease/percutaneous-nephrostomy

\section{INTECH}

open science | open minds

\author{
InTech Europe \\ University Campus STeP Ri \\ Slavka Krautzeka 83/A \\ 51000 Rijeka, Croatia \\ Phone: +385 (51) 770447 \\ Fax: +385 (51) 686166 \\ www.intechopen.com
}

\author{
InTech China \\ Unit 405, Office Block, Hotel Equatorial Shanghai \\ No.65, Yan An Road (West), Shanghai, 200040, China \\ 中国上海市延安西路65号上海国际贵都大饭店办公楼 405 单元 \\ Phone: +86-21-62489820 \\ Fax: $+86-21-62489821$
}


(C) 2012 The Author(s). Licensee IntechOpen. This is an open access article distributed under the terms of the Creative Commons Attribution 3.0 License, which permits unrestricted use, distribution, and reproduction in any medium, provided the original work is properly cited. 